\title{
Integrating Sphere Fourier Microscopy of Highly Directional Emission
}

\author{
Julia S. van der Burgt, Christian D. Dieleman, Eric Johlin, Jaco J. Geuchies, Arjan J. Houtepen,
} Bruno Ehrler, and Erik C. Garnett*

Cite This: ACS Photonics 2021, 8, 1143-1151

Read Online

\section{ACCESS | Lill Metrics \& More | 回 Article Recommendations ｜（） Supporting Information}

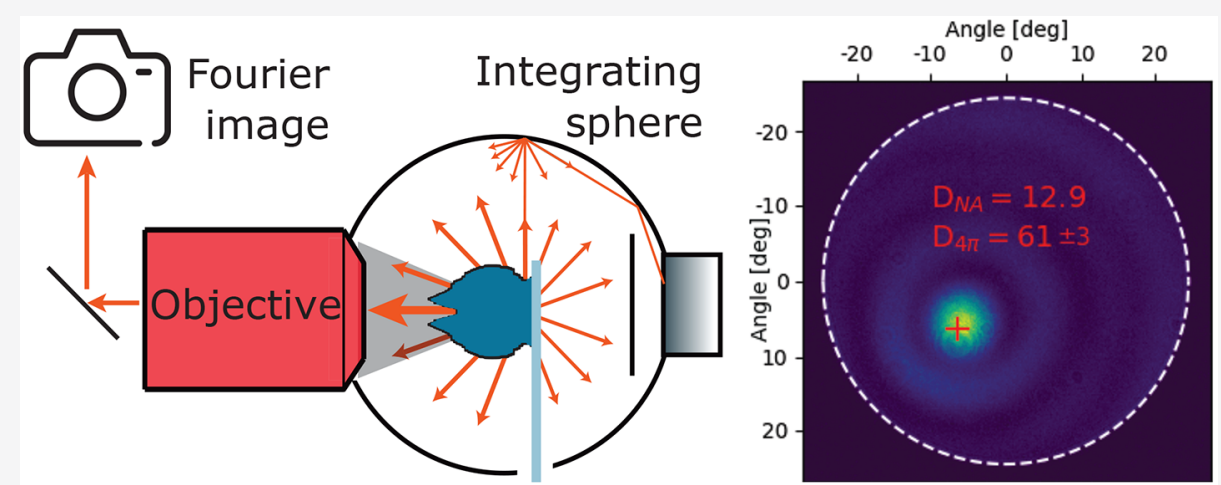

ABSTRACT: Accurately controlling light emission using nano- and microstructured lenses and antennas is an active field of research. Dielectrics are especially attractive lens materials due to their low optical losses over a broad bandwidth. In this work we measure highly directional light emission from patterned quantum dots (QDs) aligned underneath all-dielectric nanostructured microlenses. The lenses are designed with an evolutionary algorithm and have a theoretical directivity of 160 . The fabricated structures demonstrate an experimental full directivity of $61 \pm 3$, three times higher than what has been estimated before, with a beaming half-angle of $2.6^{\circ}$. This high value compared to previous works is achieved via three mechanisms. First, direct electron beam patterning of QD emitters and alignment markers allowed for more localized emission and better emitter-lens alignment. Second, the lens fabrication was refined to minimize distortions between the designed shape and the final structure. Finally, a new measurement technique was developed that combines integrating sphere microscopy with Fourier microscopy. This enables complete directivity measurements, contrary to other reported values, which are typically only partial directivities or estimates of the full directivity that rely partly on simulations. The experimentally measured values of the complete directivity were higher than predicted by combining simulations with partial directivity measurements. High directivity was obtained from three different materials (cadmium-selenide-based QDs and two lead halide perovskite materials), emitting at 520, 620, and $700 \mathrm{~nm}$, by scaling the lens size according to the emission wavelength.

KEYWORDS: directivity, nanolens, nanoantenna, dielectric, Fourier microscopy, integrating sphere microscopy

\section{INTRODUCTION}

Controlling the direction of light propagation is an important element in modern technologies. It is used in energy, lighting, and information technologies to increase device efficiency. An important metric for defining how well the light is focused in a specific direction is the directivity, borrowed from classical antenna theory. It is defined as the maximum intensity into the beaming direction, divided by the average intensity ${ }^{1}$ (eq 1 , as we will discuss in more detail there). On the macroscopic scale, light is typically directed with curved lenses and mirrors, which make use of refraction and reflection. Nanoscale structures, with sizes similar to the wavelength of light, work in a fundamentally different manner, by relying additionally on diffraction and interference effects to control the light. These effects also naturally result in wavelength selectivity, and by combining the traditional refraction/reflection effects with diffraction/interference effects the bandwidth can be adjusted. This is an important aspect in nanophotonic design. Directional light emission has been shown to increase the efficiency of LEDs, ${ }^{2-5}$ single-photon emitters, ${ }^{6,7}$ photonic circuits, ${ }^{8,9}$ and solar cells. ${ }^{10}$

Received: January 4, 2021

Published: April 9, 2021 
Table 1. Overview of Theoretical and Experimental Work on Directional Emission from (Partly) Dielectric Nanostructures

\begin{tabular}{|c|c|c|c|c|c|}
\hline & full directivity & partial directiviy (NA) & beaming half-angle & fwhm & lens (largest dimension) \\
\hline dielectric sphere (sim only) ${ }^{19}$ & 7.5 & & & & $907 \mathrm{~nm}$ \\
\hline hybrid gold nanorod/silicon nanodisk (sim only) ${ }^{22}$ & 8.5 & & & & $620 \mathrm{~nm}$ \\
\hline sphere in nitrogen vacancy centers $(\text { sim only })^{21}$ & 11 & & & & $380 \mathrm{~nm}$ \\
\hline photonic nanojet with hybrid antenna (sim only) ${ }^{23}$ & 40 & & & & $20 \mu \mathrm{m}$ \\
\hline hafnium oxide nanoantenna ${ }^{24}$ & & $18(1.49)$ & & $9^{\circ}$ & $3 \mu \mathrm{m}$ \\
\hline hybrid Yagi-Uda nanoantenna ${ }^{25}$ & 49.2 & $44.3(1.4)$ & $4.6^{\circ} / 27.3^{\circ}$ & & $728 \mathrm{~nm}$ \\
\hline InGaN/GaN quantum -well metasurfaces ${ }^{17}$ & & & $7^{\circ}$ & & $3 \mu \mathrm{m}$ \\
\hline parabolic light directors ${ }^{27}$ & & & $5.6^{\circ}$ & & $22 \mu \mathrm{m}$ \\
\hline elliptical microlenses ${ }^{28}$ & & & $1^{\circ}$ & & $16.3 \mu \mathrm{m}$ \\
\hline nanolens from EA (our previous work) ${ }^{26}$ & 22 & $18.9(0.9)$ & $3.5^{\circ}$ & & $6 \mu \mathrm{m}$ \\
\hline nanolens from EA (current work) & 61 & $12.9(0.42)$ & $2.6^{\circ}$ & $5.6^{\circ}$ & $7 \mu \mathrm{m}$ \\
\hline
\end{tabular}
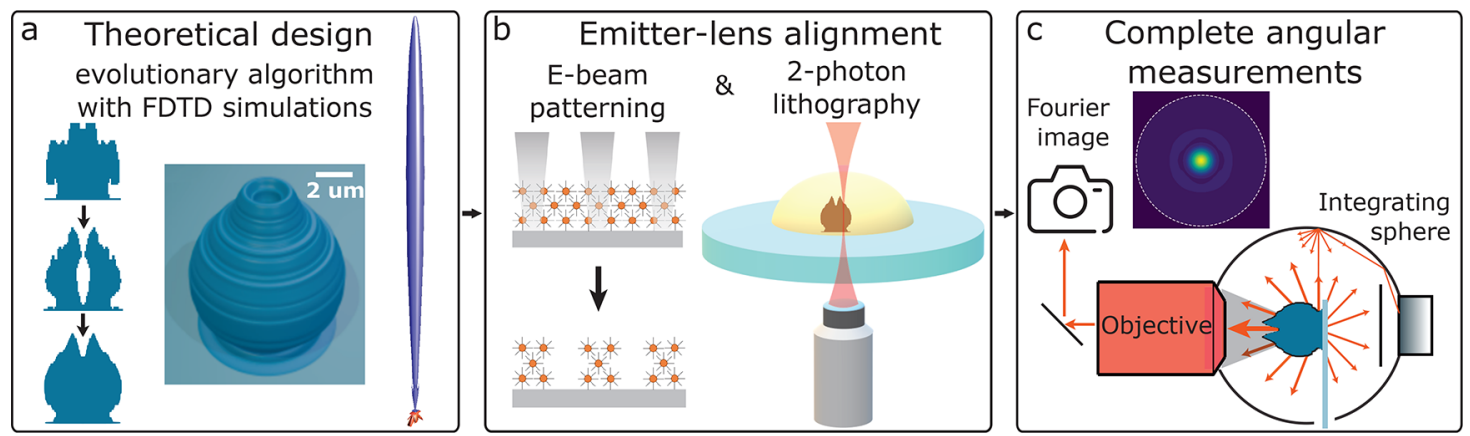

Figure 1. Schematic representation of the process toward highly directional emission. The first step (a) is the design of a 3D structure that gives directional emission from an emitter at the center. With an evolutionary algorithm, a $2 \mathrm{D}$ matrix that describes the presence of material is optimized (left). By rotating around the central axis a circularly symmetric 3D structure is obtained (center) for which the emission pattern is calculated (right). Fabrication of lenses on top of emitters consists of first patterning the emitters with direct electron-beam lithography (b, left). Upon exposure to an electron beam, the ligands in a film of CdSe-based QDs cross-link. Subsequently, unexposed parts are washed off and localized clusters remain. Next, 3D structures are fabricated from a transparent photoresist with two-photon lithography (b, right). Finally, the complete angular directivity is measured by combining Fourier microscopy with an integrating sphere (c).

Different methods have been developed to control the direction of light with nanostructures. ${ }^{11}$ Especially structures made of dielectric material are of interest, because they do not suffer from the parasitic absorption that is inherent to plasmonics. $^{12,13}$ Zone plates, which consist of concentric rings with spacings similar to the wavelength, focus light based on diffraction rather than refraction used in conventional curved lenses. ${ }^{14,15}$ Metasurfaces, with nanoscale patterns far below the wavelength of light that modify the optical phase, have been designed to make flat lenses, enhance light emission, and control its direction. ${ }^{16-18}$ Relatively simple shapes such as micro- or nanospheres can also give rise to directional emission: an emitter embedded asymmetrically in a lossless sphere can give a directivity of $7.5 .^{19}$ By placing silicon dioxide nanoparticles on a mirror, surface-enhanced Raman scattering showed an increase in enhancement factor of 250 through the combination of plasmonic and refractive effects on both excitation and emission efficiency. ${ }^{20}$ Numerical simulations have shown directivity up to 11 from nitrogen vacancy centers in diamond with all-dielectric spherical nanoantennas on top. ${ }^{21}$

To achieve higher directivity, shapes more complex than simple spheres have to be used. Ideas of more versatile nanoantenna designs used with plasmonic structures have been applied to dielectric materials as well. For a hybrid system that combines a single emitter coupled to a gold nanorod with a silicon nanodisk for redirecting the light, simulations predict a directivity of $8.5 .^{22}$ An even higher directivity of 40 (16 dB) was simulated using a photonic nanojet with a hybrid antenna consisting of a dielectric sphere and a plasmonic antenna. ${ }^{23}$
Quantum dots (QDs) in the feed gap of a hafnium oxide nanoantenna showed a partial directivity of $18(12.5 \mathrm{~dB}) .^{24} \mathrm{In}$ a hybrid system inspired by the Yagi-Uda antenna design, consisting of a gold bow-tie nanoantenna and three silicon nanorods, a directivity up to 49.2 was estimated based on a combination of experiments and simulations. ${ }^{25}$ In previous work we have reported on a method to create directional emission from gallium arsenide (GaAs) nanowires with dielectric nanostructured microlenses designed with an evolutionary algorithm (EA). ${ }^{26}$ This led to a record directivity from all-dielectric nanostructured microlenses of 22 . The given examples of theoretical and experimental work on directivity above are summarized in Table 1. Many different metrics are used to assess directivity, caused by limited experimental access to the full directivity, which complicates comparison among different works. For example, many works use beaming halfangle instead of directivity, which then ignores all power outside of the primary emission peak. Nevertheless, we have selected several relevant examples in Table 1, including metasurfaces used to direct light from $\operatorname{LEDs}^{17}$ as well as parabolic light directors ${ }^{27}$ and elliptical microlenses. ${ }^{28}$ Directivity is typically measured with Fourier microscopy, which provides the angular emission pattern only within the numerical aperture (NA) of the objective. Either the relative enhancement or partial directivity within the collection cone of the objective is reported, which strongly depends on the NA. Alternatively, the total directivity is obtained from combining measurements and simulations. 

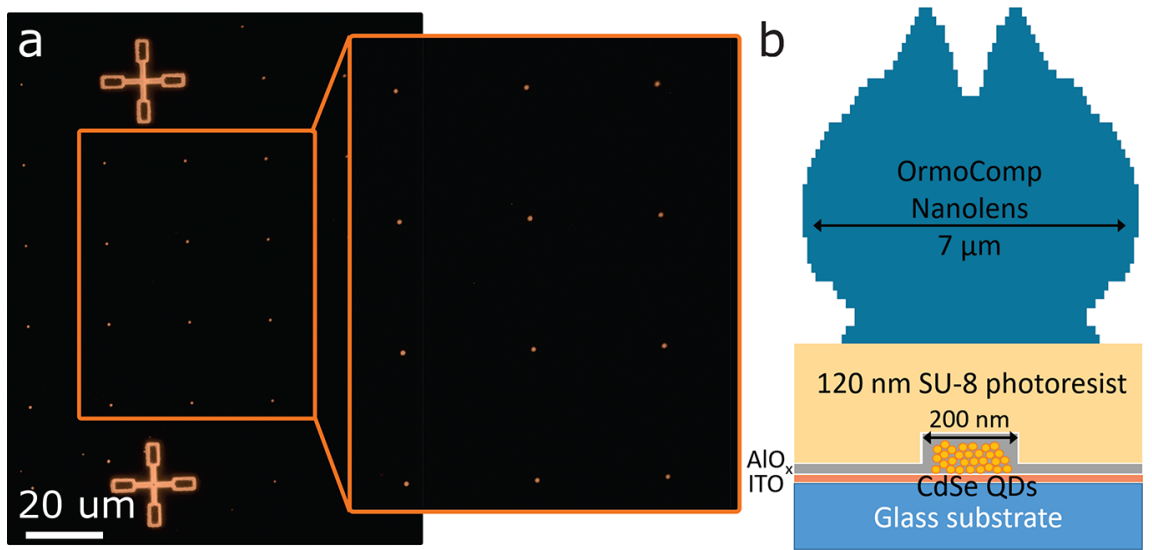

Figure 2. (a) Dark field optical microscope image of the patterned CdSe-based QDs. The crosses act as alignment markers for accurately writing the lenses on top of the small clusters. (b) Schematic of the full sample configuration. The indium tin oxide (ITO) layer provides conductivity required for e-beam patterning. The alumina $\left(\mathrm{AlO}_{x}\right)$ and SU-8 photoresist act as protection layers against the harsh conditions of the two-photon lithography process for making the nanolens. Dimensions and thicknesses are not to scale.

The directivity obtained in our previous work was also limited by experimental constraints, causing the obtained directivity to be five times lower than the value predicted by finite difference time domain (FDTD) simulations. Limited by the NA of our objective, the directivity was determined by extrapolating outside the cone of the objective and by relying on simulation results for the emission into the substrate. The randomly drop-cast nanowires limited the lens alignment precision and provided extended emitters, both reducing performance compared to an ideal point source at the lens center.

Here we show a new approach for the fabrication and characterization that has led to a 3 -fold improvement in directivity. The enhancement relies on direct electron beam patterning of quantum dot emitter arrays, which both simplifies lens alignment and provides more localized sources. The more reproducible alignment also allowed for nanoscale lens corrections in both shape and size to play a role in improved directivity, partially overcoming previous limitations. Additionally, we have developed a new technique for measuring the complete directivity, which combines a Fourier microscope with an integrating sphere. Figure 1 schematically shows the three parts of this work, which will be discussed in more detail in the following sections: the theoretical lens design with the EA, the fabrication process, and the optical directivity measurements.

\section{RESULTS AND DISCUSSION}

Evolutionary Algorithm. Although the design of nanophotonic components in some cases can be guided by analogues developed for longer wavelengths, the high material dispersion of emitter materials in the visible, combined with the interplay between interference, refraction, and diffraction effects, can complicate their efficient design. Inverse design avoids this issue, allowing simulations of a component response to directly inform the creation of new designs. Broadly, two categories of such design processes exist: gradient-based techniques and gradient-free techniques. ${ }^{29-31}$ While gradient-based approaches generally offer more computationally efficient solutions, they are inherently local optimization processes and can suffer in performance when many local optima are present. ${ }^{32}$ Evolutionary algorithms offer a conceptually simple approach to gradient-free optimization, relying on the combination of features from previous wellperforming structures to iteratively improve performance. The design here utilizes the same evolutionary process as described previously $^{26}$ to create a new directive nanolens optimized for our specific source geometry and emission frequency. In this case, we optimized for broadband and unpolarized absorption in a $100 \times 100 \times 50 \mathrm{~nm}$ volume, in the wavelength range of 470 to $570 \mathrm{~nm}$. This leads to relatively broadband directivity compared to resonant optics (Figure S1), which tend to work only over a very narrow bandwidth. At the same time, the EA still allows for optimization over a specific wavelength range, contrary to conventional macroscopic lenses, which typically act similarly over the full optical spectrum.

The as-optimized lens shows a peak in directivity at $570 \mathrm{~nm}$ emission. For optimal performance, the maximum directivity can be shifted to the emission wavelength of the emitter of choice by scaling the lens size according to the wavelength. This can be done over a range of tens to hundreds of nanometers, as long as the dispersion of the material does not change significantly in this range. By increasing the size by $8 \%$, the optimum was shifted to the emission wavelength in our experiments of $620 \mathrm{~nm}$, resulting in a theoretical directivity of 160. The final lens geometry and 3D emission profile calculated for an orientation averaged dipole are shown in Figure 1a, and the corresponding theoretical Fourier image is shown in Figure 1c. To prove the wavelength scalability, also lenses scaled for 520 and $700 \mathrm{~nm}$ emission were investigated, giving a directivity of 152 and 146, respectively. The small variations in directivity for the different wavelengths are within the accuracy of the simulations caused by finite meshing.

Fabrication and Measurement Techniques. An important aspect for optimizing the performance is the position and spatial extent of the emitters with respect to the lens. The lens performs best for a point source at the center of the lens; thus emitters should be fabricated that most closely resemble this. In earlier work, misalignment and large emitter size were the major causes for a reduction in performance. ${ }^{26}$ For this work, we decided to use cadmium selenide/cadmium sulfide/ zinc sulfide (CdSe/CdS/ZnS) QDs, since they are efficient and stable emitters. With a recently developed method, these QDs can be patterned in any design of choice with direct electron-beam (e-beam) lithography, ${ }^{33}$ shown schematically on the left of Figure $1 \mathrm{~b}$ and experimentally in Figure 2a. A spin- 


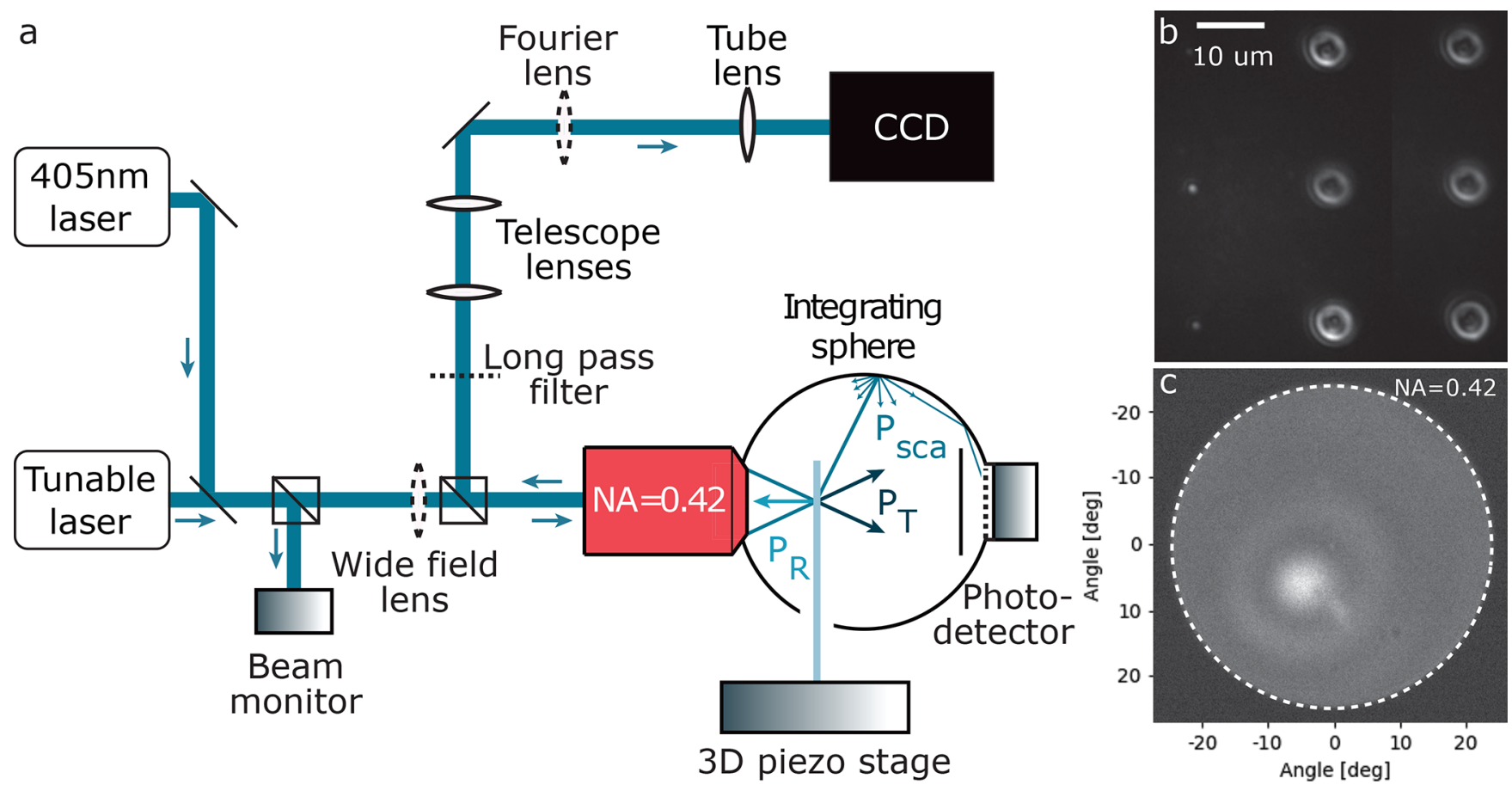

Figure 3. (a) Schematic representation of the combined integrating sphere and Fourier microscope for complete angular measurement of directivity. By moving out the Fourier lens, the sample is seen in real space to find the structures. Measurements are taken in the Fourier configuration with $450 \mathrm{~nm}$ long-pass filters to remove the $405 \mathrm{~nm}$ laser excitation, where the CCD gives the angular emission pattern in the cone of the objective, while the integrating sphere photodetector collects light emitted in all other directions. The tunable laser is used to determine the collection efficiencies at the emission wavelength and the beam monitor tracks fluctuations in excitation intensity. With the sample mounted on a piezo stage the exact position of maximum directivity can be found. (b) Real space image of bare clusters (left column) and lenses (right two columns) in wide-field laser illumination. (c) Fourier image of lenses in wide-field laser illumination.

coated film of QDs is locally exposed to an electron beam, which causes cross-linking of the oleate ligands and makes the QDs insoluble. Subsequently the sample is developed by redispersing the unexposed QDs in tetrahydrofuran, such that only the exposed QDs remain on the indium-tin-oxide (ITO)-coated substrate. This allowed us to make a square array of CdSe-based QD clusters of $200 \mathrm{~nm}$ in diameter and 50 $\mathrm{nm}$ in height (Figure S3), spaced $20 \mu \mathrm{m}$ apart, and we included alignment markers for placing the lenses, as shown in Figure 2a.

The nanolens is fabricated from a transparent photoresist, through two-photon lithography. ${ }^{34}$ In the fabrication process the sample is exposed to high-intensity infrared laser illumination and different chemicals. To shield the QDs from these harsh conditions, two protection layers are applied. First, $15 \mathrm{~nm}$ of alumina is evaporated on the sample to protect the QDs from chemicals and to bind the clusters more strongly on the substrate. Subsequently, a $120 \mathrm{~nm}$ film of transparent SU-8 photoresist is spin coated on the sample. This layer acts as a spacer layer, such that the QDs are not directly in the focus of the laser during the lithography process. ${ }^{26}$ The complete configuration of the sample, with all layers and materials present, is shown in Figure $2 b$.

Directivity is defined as the maximum intensity $\left(p_{\max }\right)$ divided by the average intensity in all directions $\left(p_{\text {av }}\right)$, i.e., the total emission $\left(P_{\text {tot }}\right)$ into the $4 \pi$ solid angle of a sphere divided by $4 \pi:^{1}$

$$
D=\frac{p_{\max }}{p_{\mathrm{av}}}=\frac{4 \pi p_{\max }}{P_{\mathrm{tot}}}
$$

The conventional way to measure directivity is with a Fourier microscope. In these measurements, only light emitted into the cone of the objective is detected, from which the partial directivity within the numerical aperture (NA) of the objective can be determined. For air objectives (oil immersion would alter the performance of the lens), which have a maximum NA of $\sim 0.95$, a maximum $69 \%$ of the forward hemisphere is collected and none of the light emitted in the backward direction. Even with two perfect objectives (one on each side of the substrate), the substantial fraction of light that is waveguided in the substrate and either scattered out or emitted at the edges will be lost. Previous works on directivity only report the partial directivity ${ }^{24}$ or rely partly on simulations for determining the full directivity. ${ }^{25}$ An alternative to Fourier imaging for wide-angle measurements is to place a detector on a rotation stage and scan in a circle at a fixed angle. ${ }^{35}$ However, using such an approach for complete directivity would be very time-consuming and complex, as it would require very accurate scanning over the full three-dimensional sphere. For individual nano- and microstructured lenses the signal is too low for such an approach to be feasible. Due to the difficulties above, experimental measurements of the full directivity are absent from the literature.

We built a setup with which we can obtain the full directivity of an individual nanostructured microlens coupled to a $200 \mathrm{~nm}$ emitter patch in a single measurement. To achieve this, we combined a Fourier microscope with an integrating sphere (IS), shown schematically in Figure 3a. The integrating sphere gives the missing piece of information in the Fourier measurement, by collecting all the light that was emitted outside the cone of the objective. This light is scattered around 
in the IS and measured with a photodetector on the back, while at the same time an image is taken with a CCD camera in the Fourier plane of the microscope. The Fourier image provides $p_{\max }$ and by combining the Fourier and IS signals $P_{\text {tot }}$ can be determined. Combining these two signals demands careful calibration of the collection efficiencies; a detailed description of the required reference measurements and the full calculation can be found in the Supporting Information. With proper calibration, our integrating sphere Fourier microscopy method can therefore provide an accurate full directivity value for any structure with an emission peak that falls within the NA of the objective lens.

The sample is mounted on a piezo stage, which allows us to scan the focused excitation spot through the structure in three dimensions and find the position of highest directivity. Here we benefit from the fact that the directivity can be determined in a single measurement, allowing us to take detailed maps over the lenses. After finding the optimal position, repeated measurements were taken on this fixed location, to determine the noise on the signals and get accurate directivity values. With an additional lens in front of the objective, we can create full field laser illumination to excite multiple lenses. We can find the structures in a real space image (Figure $3 \mathrm{~b}$ ), and by inserting the Fourier lens, Fourier images can be taken for either full field illumination (Figure 3c) or focused excitation (during the measurements). Note that for combining the Fourier microscope with an integrating sphere, an objective with a long working distance of $17 \mathrm{~mm}$ has to be used, which leads to a relatively low NA of 0.42 . As a consequence, our measured partial directivities might appear relatively low. As long as the peak of maximum intensity lies within the cone of the objective, this low NA is not a problem for the determination of full directivity.

With nanoscale corrections we optimized the fabrication of the nanolenses, to make them closely resemble the designed structure. We optimized two aspects separately: the size and the shape. Due to slight shrinking of the photoresist in the development process, structures made with two-photon lithography typically have to be written with slightly larger coordinates in order to end up with the correct size. The amount of shrinking depends on the laser power used for writing (see Figure S4) and the density of points with which the structure is written. Lenses with different size scaling were fabricated and measured with a scanning electron microscope (SEM). The diameter of the lens is easily measured from the top view. The height of the lens was checked by cutting a cross section through a lens with focused ion beam (FIB) milling. To be less sensitive to the exact location of the FIB cutting plane, we fabricated elongated lenses with a fixed cross section over several micrometers, which is why the lenses shown in Figure 4 are not circularly symmetric.

Higher laser power typically gives sharper features, but when the intensity is too high, this results in small explosions in the photoresist (Figure S5). ${ }^{36,37}$ Therefore, we fine-tuned the exact shape by comparing the FIB cross sections with the desired design. Figure $4 a$ shows the first step, in which the lens was written based on the original binary matrix from the EA together with the desired cross section in orange. Subsequently, pixels corresponding to regions with too much material (marked red and orange in Figure 4b) are removed from the writing coordinates. This first optimization resulted in a close resemblance between the observed cross section and the desired shape (Figure 4c). In the second optimization the

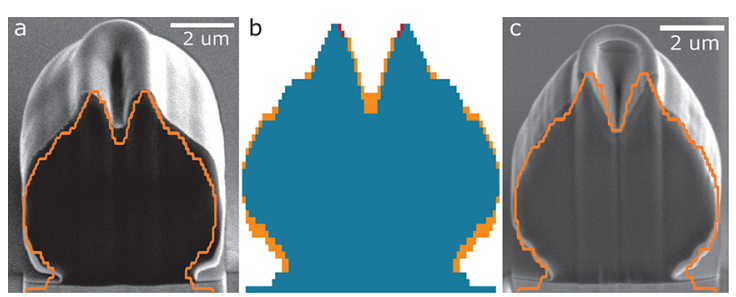

Figure 4. Optimizing the lens shape. (a) Lens based on the original binary matrix, with too much material at certain locations compared to the desired shape (orange line). (b) Original binary matrix (all pixels), the pixels that were removed in the first optimization (red and orange), resulting in the lens in (c). and the pixels that were added again in the last optimization (red).

two red pixels in the top are added again to better match the shape of the top of the lens.

Complete Angular Directivity Measurements. With the techniques and optimizations described above, we were able to accurately fabricate nanolenses on top of arrays of CdSe-based QD clusters, as shown in Figure 5a. The first directivity measurements were performed on lenses from the original design, like the one in Figure 4a. The writing coordinates were scaled by $102 \%, 106 \%$, and $110 \%$ to find the optimum size. These lenses all performed rather poorly, with a partial directivity, i.e., only in the NA of the objective $\left(D_{\mathrm{NA}}\right)$, between 3 and 4 and a total directivity $\left(D_{4 \pi}\right)$ between 8 and 26 (Figure 5d left and Table S1). The first optimization again consisted of lenses in the three different sizes. The adjustment in shape turned out to be an essential step: a partial directivity as high as $D_{\mathrm{NA}}=10.9$ was observed, corresponding to a total directivity of $D_{4 \pi}=41$. All five lenses of scale $102 \%$ performed similarly; see Figure 5d middle and Table S2 for all results. The last optimization consisted of a detailed size tuning and three small variations in the design. The sizes were varied between $98 \%$ and $106 \%$ in steps of $2 \%$. The three designs were the design of optimization 1 (thus red and orange pixels in Figure $4 \mathrm{~b}$ removed), the design of optimization 2 , i.e., only the orange pixels removed, and this same design, but elongated in height by $2 \%$, because SEM analysis showed that the previous set of lenses was slightly too flat.

In this second optimization we found the highest partial directivity of $D_{\mathrm{NA}}=12.9$ and a beaming half-angle $(\sigma)$ of $2.6^{\circ}$, of which the Fourier image is shown in Figure $5 b$ (for reference of bare emitters without a lens on top, see Figure S6). Repeated measurements of the same position were taken to accurately determine the total directivity and the uncertainty on this value, resulting in an average $D_{\mathrm{NA}}=12.1 \pm 0.1$ and $D_{4 \pi}$ $=61 \pm 3$ over 10 measurements. Repeated measurement over a longer time show a decrease in directivity, as plotted in Figure 5c lower panel. We attribute this to degradation of the QDs, since also the signal intensity of both detectors decreases over time (Figure S7), and degradation was also observed for emitters without a lens on top (Figure S8). On the other four best performing lenses, 18 repeated measurements were taken, resulting in $D_{\mathrm{NA}}$ and $D_{4 \pi}$ values as plotted on the right of Figure $5 \mathrm{~d}$. More measurements for determining the accuracy and the effect of excitation power on multiple lenses can be found in Figure S9. Results of the 15 best performing lenses, out of the 48 lenses that were fabricated and measured, are summarized in Table S3. From this analysis we can conclude that we have fabricated at least 15 lenses with $D_{4 \pi}>45$, with probably even better performance before degradation. All 

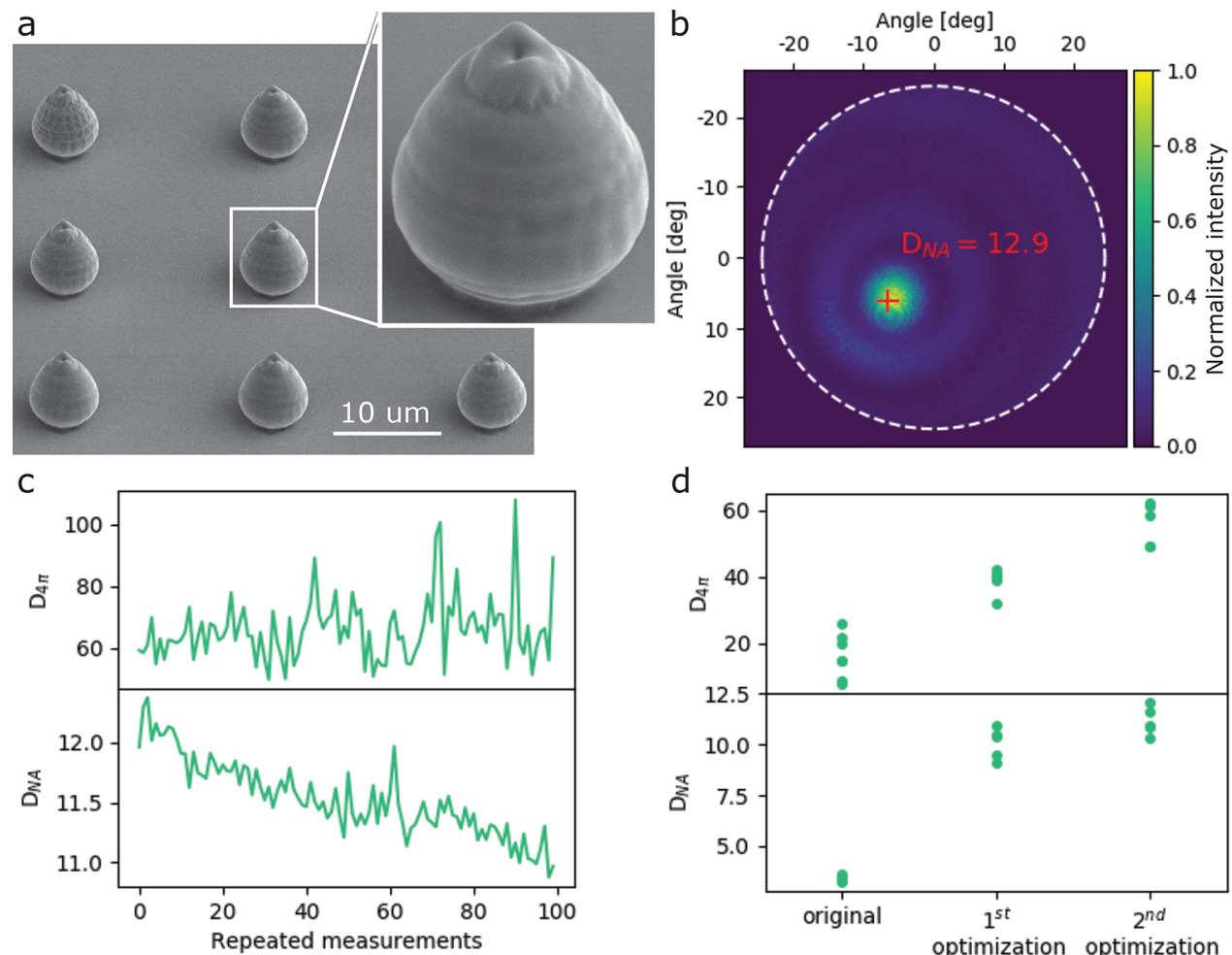

Figure 5. Results of the complete process. (a) Nanolenses accurately written on an array of CdSe-based QDs clusters. (b) Fourier image of the lens with highest measured directivity, of $D_{\mathrm{NA}}=12.9$. (c) Repeated measurements on a fixed location show degradation of the lens and/or emitter, causing a lowering in $D_{\mathrm{NA}}$ (lower panel) and an increase in the noise on $D_{4 \pi}$ (top panel). (d) Results of the best performing lenses of each of the optimization steps.

lenses with a scaling of $106 \%$ performed similarly, and no trend could be observed between the different designs in the final optimization step. Also each of the other size scales had one or two high-performing lenses.

As a proof of concept of the wavelength scalability, we fabricated lenses scaled in size for two different wavelengths: 520 and $700 \mathrm{~nm}$, the emission wavelengths of cesium lead bromide $\left(\mathrm{CsPBr}_{3}\right)$ perovskite nanocrystals and a methylammonium lead halide $\left(\mathrm{MAPbBr}_{0.2} \mathrm{I}_{0.8}\right)$ thin film, respectively. These samples consisted of a continuous film of emitters instead of localized clusters, and only the partial directivity $D_{\mathrm{NA}}$ could be determined. Even with these limitations the results look promising: both systems reach $D_{\mathrm{NA}}>11$; Fourier images are shown in Figure S10. This result is surprisingly good for lenses on a continuous film, where no localized emitters were created. However, these halide perovskites are known for their photobrightening. ${ }^{38}$ We hypothesize that focusing of the excitation laser by the lens causes local photobrightening in the film, resulting in more localized emission. We have performed optical simulations showing that the fwhm of the excitation spot size created by the nanostructured microlens is approximately $200 \mathrm{~nm}$ (Figure S11) and would lead to a directivity of 200, consistent with the patch size and theoretical directivity of the localized CdSe emitters. This very interesting observation requires further investigation in future work and may enable a self-aligned mechanism for greatly improved performance.

From the large increase in directivity by adjusting the design, we can conclude that the directivity is sensitive to the exact shape of the lens structure. The small variations in the final optimization did not show any significant trend, indicating that lens to lens variation due to fabrication inaccuracy is larger than the designed adjustments in this final step. The dependence of the directivity on the exact shape matches well with results from simulations, where we also noted a large sensitivity to the shape of the structures. Since it is impossible to have perfect agreement between the simulated and the fabricated structures, combining experimental results with simulations will be inaccurate. This shows the importance of our fully experimental measurement, which allows us to investigate the actual fabricated structures without relying partially on simulations. For a cluster of emitters with a diameter of $200 \mathrm{~nm}$, simulations predict a total directivity of $D_{4 \pi \text {,theory }}=131$ and $D_{\mathrm{NA} \text {,theory }}=35$. Although our measured $D_{\mathrm{NA}, \text { experiment }}=12$ is roughly three times lower compared to theoretical performance, our measured $D_{4 \pi \text {,experiment }}=61$ is almost half the theoretical value and thus better than what would be predicted by extrapolating $D_{\mathrm{NA} \text {,experiment }}$.

We assign the fact that the measured directivity is still significantly lower than the theoretical directivity to fabrication errors, primarily alignment errors. Simulations show that the directivity is decreased by $50 \%$ if the emitter is misaligned by only $130 \mathrm{~nm}$ with respect to the center of the lens (Figure S13a). The tilting of the emission peak from the normal is also consistent with a misalignment of approximately $200 \mathrm{~nm}$ (Figure S13). Also small size variations might still play a role, as we predict a drop in directivity of $20 \%$ for each $3 \%$ offset in size (Figure S13b). In our last optimization step, the lenses with a size of $106 \%$ performed best, but each of the other sizes also had a positive outlier. This probably corresponds to occasionally very well aligned lenses, such that they perform well despite their slight size offset. We ascribe the different optimal size found in the last two optimization steps (102\% vs $106 \%)$ to variation in sample absorbance. Since the resulting 
size of structures made by two-photon lithography strongly depends on the laser power, a slightly higher absorption in the sample requires larger writing settings in order to obtain the same size.

\section{CONCLUSION}

With accurate nanoscale optimization of the fabrication technique, we have achieved a record in directivity from alldielectric nanolenses, three times higher than the previously reported value. We have fully experimentally determined a maximum total directivity of $D_{4 \pi}=61 \pm 3$, corresponding to a partial directivity within the cone of the objective of $D_{\mathrm{NA}}=$ $12.1 \pm 0.1$. At low laser power excitation, at which only $D_{\mathrm{NA}}$ could be accurately determined, partial directivity values up to $12.9 \pm 0.1$ were observed. This shows that with accurate fabrication techniques, highly directional emission from CdSebased QDs can be obtained with all-dielectric nanostructures. An essential step to achieve this was the patterning of emitters with direct e-beam lithography into an ordered array of small clusters with alignment markers, such that an array of lenses could be accurately fabricated on top of the emitters. The combination of an integrating sphere and a Fourier microscope allowed for complete angular measurement of the directivity, which was $35 \%$ higher than what would have been predicted by a combination of Fourier microscopy and simulations. With the knowledge obtained here, we can take further steps in directional emission with dielectric nanostructures. This work was focused on single nanolenses. The capability of creating patterned emitters opens up possibilities for future work in which one could make use of array effects on top of the individual nanolens effects or design periodic structures that provide directivity.

\section{EXPERIMENTAL METHODS}

Fabrication. All samples were fabricated on ITO-coated glass coverslips of thickness $170 \mu \mathrm{m}$ with a resistance of $8-12$ ohms per square from Diamond Coatings. Substrates were cleaned before spin coating the quantum dot layer by $15 \mathrm{~min}$ of sonication in demineralized water, acetone, and isopropanol, followed by $15 \mathrm{~min}$ of oxygen plasma treatment. CdSe-based QDs were fabricated according to an adjusted protocol ${ }^{39-41}$ described in detail in the Supporting Information. QDs dispersed in toluene were spin coated at $500 \mathrm{rpm}$ for $90 \mathrm{~s}$ followed by $30 \mathrm{~s}$ at $1000 \mathrm{rpm}$. These films were exposed in a Raith Voyager commercial e-beam lithography system with an accelerating voltage of $50 \mathrm{kV}$, in MC40 or MC60 column mode with a $0.94 \pm 0.02$ or $2.20 \pm 0.05 \mathrm{nA}$ beam current, respectively. Samples were developed in tetrahydrofuran for 30 $\mathrm{s}$, followed by a 5 s rinse in anhydrous $n$-octane, before drying with nitrogen. The alumina layer was applied with an electron beam physical vapor deposition system from Polyteknik. A source of $\mathrm{Al}_{2} \mathrm{O}_{3}$ was used with additional oxygen inflow to evaporate $15 \mathrm{~nm}$ of alumina $\left(\mathrm{AlO}_{x}\right)$ of unknown composition at a rate of $0.2 \mathrm{~nm} / \mathrm{s}$. The spacer layer of transparent SU-8 photoresist was made by spin coating $120 \mu \mathrm{L}$ of $1: 10$ diluted SU-8/cyclopentanone at $4000 \mathrm{rpm}$ for $60 \mathrm{~s}$. Subsequently the samples were dried on a hot plate at $100{ }^{\circ} \mathrm{C}$ for $60 \mathrm{~s}$, exposed with a hand-held UV lamp at $365 \mathrm{~nm}$ for $10 \mathrm{~min}$, and further hardened with a final hard bake on the hot plate of again 100 ${ }^{\circ} \mathrm{C}$ for 60 s. The lenses are fabricated with two-photon lithography (Photonic Pro, Nanoscribe GmbH) from undiluted OrmoComp photoresist (Micro Resist Technology
$\mathrm{GmbH}$ ), which has low fluorescence and high transparency and stability. ${ }^{42}$ The alignment markers were used for positioning the lenses. In case the actual clusters of emitters could be seen in the Nanoscribe microscope (ca. 30\% of the time), small manual adjustments were made to the exact position of the lens. The structures are developed with mr-Dev 600 (Micro Resist Technology $\mathrm{GmbH}$ ) for $25 \mathrm{~min}$, followed by $5 \mathrm{~min}$ in isopropanol. During the rinsing in isopropanol, the structures are exposed to $365 \mathrm{~nm} \mathrm{UV} \mathrm{light} \mathrm{to} \mathrm{make} \mathrm{the} \mathrm{lenses}$ more robust. ${ }^{43}$ For characterization with SEM, lenses were sputter-coated with $5 \mathrm{~nm}$ chrome and $15 \mathrm{~nm}$ gold conductive layers. Focused ion beam milling for making cross cuts and SEM imaging were done on a FEI Helios Nanolab 600.

Measurement Setup. The combined Fourier microscope and integrating sphere setup was created by adding a Fourier microscope to an existing integrating sphere setup, which has been described before. ${ }^{44}$ The Fourier microscope was added by placing a pellicle beam splitter (BP145B1 Thorlabs $\mathrm{GmbH}$ ) right behind the objective. Excitation was done with a $405 \mathrm{~nm}$ laser (S1FC405 Thorlabs $\mathrm{GmbH}$ ). The light goes via a longpass filter at $450 \mathrm{~nm}$, a set of telescopic lenses, a Fourier lens that can be taken out for real-space imaging, and a tube lens to the CCD camera (Retiga Lumo model 01-RET-LUMO-R-M16-C, Teledyne Photometrics). Fourier images were taken at 200 and $500 \mathrm{~ms}$ integration time, with $2 \times 2$ pixel binning.

\section{ASSOCIATED CONTENT}

\section{Supporting Information}

The Supporting Information is available free of charge at https://pubs.acs.org/doi/10.1021/acsphotonics.1c00010.

Details of directivity calculation and additional data (PDF)

\section{AUTHOR INFORMATION}

\section{Corresponding Author}

Erik C. Garnett - AMOLF Institute, 1098XG Amsterdam, The Netherlands; 10 orcid.org/0000-0002-9158-8326; Phone: 0031207547100; Email: E.garnett@amolf.nl

\section{Authors}

Julia S. van der Burgt - AMOLF Institute, 1098XG Amsterdam, The Netherlands

Christian D. Dieleman - AMOLF Institute, 1098XG Amsterdam, The Netherlands; Advanced Reseach Center for Nanolithography, 1098XG Amsterdam, The Netherlands; (1) orcid.org/0000-0002-6571-7657

Eric Johlin - Nanophotonic Energy Materials, Western Engineering, Western University, SEB 3094 London, Canada; (1) orcid.org/0000-0002-2463-6252

Jaco J. Geuchies - Optoelectronic Materials, Faculty of Applied Sciences, Delft University of Technology, 2629HZ Delft, The Netherlands; (1) orcid.org/0000-0002-0758-9140

Arjan J. Houtepen - Optoelectronic Materials, Faculty of Applied Sciences, Delft University of Technology, 2629HZ Delft, The Netherlands; $\odot$ orcid.org/0000-0001-8328443X

Bruno Ehrler - AMOLF Institute, 1098XG Amsterdam, The

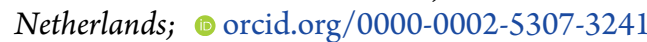

Complete contact information is available at: https://pubs.acs.org/10.1021/acsphotonics.1c00010 


\section{Notes}

The authors declare no competing financial interest.

\section{ACKNOWLEDGMENTS}

This work is part of the research program Mat4Sus, which is financed by The Netherlands Organisation for Scientific Research (NWO). The work has been carried out at Amolf, an NWO-funded institute. A.J.H. and J.J.G. gratefully acknowledge financial support from the European Research Council Horizon 2020 ERC Grant Agreement No. 678004.

\section{REFERENCES}

(1) Novotny, L.; Hecht, B. Principles of Nano-Optics; Cambridge University Press: Cambridge, 2009.

(2) Lozano, G.; Louwers, D. J.; Rodríguez, S. R. K.; Murai, S.; Jansen, O. T. A.; Verschuuren, M. A.; Gómez Rivas, J. Plasmonics for solid-state lighting: Enhanced excitation and directional emission of highly efficient light sources. Light: Sci. Appl. 2013, 2 (1-7), No. e66.

(3) Gorsky, S.; Zhang, R.; Gok, A.; Wang, R.; Kebede, K.; Lenef, A.; Raukas, M.; Dal Negro, L. Directional light emission enhancement from LED-phosphor converters using dielectric Vogel spiral arrays. APL Photonics 2018, 3 (1-12), 126103.

(4) Gorsky, S.; Britton, W. A.; Chen, Y.; Montaner, J.; Lenef, A.; Raukas, M.; Dal Negro, L. Engineered hyperuniformity for directional light extraction. APL Photonics 2019, 4 (1-13), 110801.

(5) Khaidarov, E.; Liu, Z.; Paniagua-Domínguez, R.; Ha, S. T.; Valuckas, V.; Liang, X.; Akimov, Y.; Bai, P.; Png, C. E.; Demir, H. V.; Kuznetsov, A. I. Control of LED Emission with Functional Dielectric Metasurfaces. Laser Photonics Rev. 2020, 14, 1-8.

(6) Koenderink, A. F. Single-Photon Nanoantennas. ACS Photonics 2017, 4, 710-722.

(7) Sun, Y.; Yaroshenko, V.; Chebykin, A.; Ageev, E.; Makarov, S.; Zuev, D. Metal-dielectric nanoantenna for radiation control of a single-photon emitter. Opt. Mater. Express 2020, 10, 29-35.

(8) Yang, H. Y. D.; Alexopoulos, N. G.; Yablonovitch, E. Photonic band-gap materials for high-gain printed circuit antennas. IEEE Trans. Antennas Propag. 1997, 45, 185-187.

(9) Li, Z.; Aydin, K.; Ozbay, E. Highly directional emission from photonic crystals with a wide bandwidth. Appl. Phys. Lett. 2007, 91, 89-92.

(10) Van Der Burgt, J. S.; Garnett, E. C. Nanophotonic Emission Control for Improved Photovoltaic Efficiency. ACS Photonics 2020, 7, 1589-1602.

(11) Novotny, L.; Van Hulst, N. Antennas for light. Nat. Photonics 2011, 5, 83-90.

(12) Krasnok, A. E.; Miroshnichenko, A. E.; Belov, P. A.; Kivshar, Y. S. All-dielectric optical nanoantennas. Opt. Express 2012, 20, 2059920604.

(13) Decker, M.; Staude, I. Resonant dielectric nanostructures: A low-loss platform for functional nanophotonics. J. Opt. (Bristol, U. K.) 2016, 18, 1-31.

(14) Kim, H. C.; Ko, H.; Cheng, M. High efficient optical focusing of a zone plate composed of metal/dielectric multilayer. Opt. Express 2009, 17, 3078-3083.

(15) Lu, B. R.; Li, J. X.; Guo, H. B.; Gao, C.; Huq, E.; Qu, X. P.; Chen, Y.; Liu, R. Dielectric Fresnel zone plates on optical fibers for micro-focusing applications. Microelectron. Eng. 2011, 88, 2650-2652.

(16) Vaskin, A.; Kolkowski, R.; Koenderink, A. F.; Staude, I. Lightemitting metasurfaces. Nanophotonics 2019, 8, 1151-1198.

(17) Iyer, P. P.; DeCrescent, R. A.; Mohtashami, Y.; Lheureux, G.; Butakov, N. A.; Alhassan, A.; Weisbuch, C.; Nakamura, S.; DenBaars, S. P.; Schuller, J. A. Unidirectional luminescence from InGaN/GaN quantum-well metasurfaces. Nat. Photonics 2020, 14, 543-548.

(18) Huang, T. Y.; Grote, R. R.; Mann, S. A.; Hopper, D. A.; Exarhos, A. L.; Lopez, G. G.; Garnett, E. C.; Bassett, L. C. A monolithic immersion metalens for imaging solid-state quantum emitters. Nat. Commun. 2019, 10 (1-8), 2392.
(19) Mann, S. A.; Grote, R. R.; Osgood, R. M.; Alù, A.; Garnett, E. C. Opportunities and Limitations for Nanophotonic Structures to Exceed the Shockley-Queisser Limit. ACS Nano 2016, 10, 86208631.

(20) Kamp, M.; de Nijs, B.; Kongsuwan, N.; Saba, M.; Chikkaraddy, R.; Readman, C. A.; Deacon, W. M.; Griffiths, J.; Barrow, S. J.; Ojambati, O. S.; Wright, D.; Huang, J.; Hess, O.; Scherman, O. A.; Baumberg, J. J. Cascaded nanooptics to probe microsecond atomicscale phenomena. Proc. Natl. Acad. Sci. U. S. A. 2020, 117, 1481914826.

(21) Krasnok, A. E.; Maloshtan, A.; Chigrin, D. N.; Kivshar, Y. S.; Belov, P. A. Enhanced emission extraction and selective excitation of NV centers with all-dielectric nanoantennas. Laser and Photonics Reviews 2015, 9, 385-391.

(22) Rusak, E.; Staude, I.; Decker, M.; Sautter, J.; Miroshnichenko, A. E.; Powell, D. A.; Neshev, D. N.; Kivshar, Y. S. Hybrid nanoantennas for directional emission enhancement. Appl. Phys. Lett. 2014, 105 (1-5), 221109.

(23) Bonakdar, A.; Mohseni, H. Hybrid optical antenna with high directivity gain. Opt. Lett. 2013, 38, 2726-2728.

(24) Peter, M.; Hildebrandt, A.; Schlickriede, C.; Gharib, K.; Zentgraf, T.; Förstner, J.; Linden, S. Directional Emission from Dielectric Leaky-Wave Nanoantennas. Nano Lett. 2017, 17, 41784183.

(25) Ho, J.; Fu, Y. H.; Dong, Z.; Paniagua-Dominguez, R.; Koay, E. H.; Yu, Y. F.; Valuckas, V.; Kuznetsov, A. I.; Yang, J. K. Highly directive hybrid metal-dielectric yagi-uda nanoantennas. ACS Nano 2018, 12, 8616-8624.

(26) Johlin, E.; Mann, S. A.; Kasture, S.; Koenderink, A. F.; Garnett, E. C. Broadband highly directive 3D nanophotonic lenses. Nat. Commun. 2018, 9 (1-8), 4742.

(27) Atwater, J. H.; Spinelli, P.; Kosten, E.; Parsons, J.; Van Lare, C.; Van De Groep, J.; Garcia De Abajo, J.; Polman, A.; Atwater, H. A. Microphotonic parabolic light directors fabricated by two-photon lithography. Appl. Phys. Lett. 2011, 99 (1-3), 151113.

(28) Bogucki, A.; Zinkiewicz, Ł.; Grzeszczyk, M.; Pacuski, W.; Nogajewski, K.; Kazimierczuk, T.; Rodek, A.; Suffczyński, J.; Watanabe, K.; Taniguchi, T.; Wasylczyk, P.; Potemski, M.; Kossacki, P. Ultra-long-working-distance spectroscopy of single nanostructures with aspherical solid immersion microlenses. Light: Sci. Appl. 2020, 9 (1-11), 48.

(29) Molesky, S.; Lin, Z.; Piggott, A. Y.; Jin, W.; Vucković, J.; Rodriguez, A. W. Inverse design in nanophotonics. Nat. Photonics 2018, 12, 659-670.

(30) Jensen, J. S.; Sigmund, O. Topology optimization for nanophotonics. Laser and Photonics Reviews 2011, 5, 308-321.

(31) Lu, J.; Vučković, J. Nanophotonic computational design. Opt. Express 2013, 21, 13351-13367.

(32) Yu, S.; Wang, C.; Sun, C.; Chen, W. Topology optimization for light-trapping structure in solar cells. Structural and Multidisciplinary Optimization 2014, 50, 367-382.

(33) Dieleman, C. D.; Ding, W.; Wu, L.; Thakur, N.; Bespalov, I.; Daiber, B.; Ekinci, Y.; Castellanos, S.; Ehrler, B. Universal direct patterning of colloidal quantum dots by (extreme) ultraviolet and electron beam lithography. Nanoscale 2020, 12, 11306-11316.

(34) Gissibl, T.; Thiele, S.; Herkommer, A.; Giessen, H. Twophoton direct laser writing of ultracompact multi-lens objectives. Nat. Photonics 2016, 10, 554-560.

(35) Neder, V.; Ra'Di, Y.; Alù, A.; Polman, A. Combined Metagratings for Efficient Broad-Angle Scattering Metasurface. ACS Photonics 2019, 6, 1010-1017.

(36) Mueller, J. B.; Fischer, J.; Mange, Y. J.; Nann, T.; Wegener, M. In-situ local temperature measurement during three-dimensional direct laser writing. Appl. Phys. Lett. 2013, 103 (1-4), 123107.

(37) Fischer, J.; Mueller, J. B.; Kaschke, J.; Wolf, T. J. A.; Unterreiner, A.-N.; Wegener, M. Three-dimensional multi-photon direct laser writing with variable repetition rate. Opt. Express 2013, 21, 26244-26260. 
(38) Andaji-Garmaroudi, Z.; Anaya, M.; Pearson, A. J.; Stranks, S. D. Photobrightening in Lead Halide Perovskites: Observations, Mechanisms, and Future Potential. Adv. Energy Mater. 2020, 10, 1-12.

(39) Hanifi, D. A.; Bronstein, N. D.; Koscher, B. A.; Nett, Z.; Swabeck, J. K.; Takano, K.; Schwartzberg, A. M.; Maserati, L.; Vandewal, K.; van de Burgt, Y.; Salleo, A.; Alivisatos, A. P. Redefining near-unity luminescence in quantum dots with photothermal threshold quantum yield. Science 2019, 363, 1199-1202.

(40) Chen, O.; Zhao, J.; Chauhan, V. P.; Cui, J.; Wong, C.; Harris, D. K.; Wei, H.; Han, H. S.; Fukumura, D.; Jain, R. K.; Bawendi, M. G. Compact high-quality $\mathrm{CdSe}-\mathrm{CdS}$ core-shell nanocrystals with narrow emission linewidths and suppressed blinking. Nat. Mater. 2013, 12, 445-451.

(41) Boldt, K.; Kirkwood, N.; Beane, G. A.; Mulvaney, P. Synthesis of highly luminescent and photo-stable, graded shell CdSe $/ \mathrm{Cd}_{x} \mathrm{Zn}_{1-x} \mathrm{~S}$ nanoparticles by in situ alloying. Chem. Mater. 2013, 25, 4731-4738. (42) Sikanen, T.; Aura, S.; Heikkilä, L.; Kotiaho, T.; Franssila, S.; Kostiainen, R. Hybrid ceramic polymers: New, nonbiofouling, and optically transparent materials for microfluidics. Anal. Chem. 2010, 82, 3874-3882.

(43) Purtov, J.; Verch, A.; Rogin, P.; Hensel, R. Improved development procedure to enhance the stability of microstructures created by two-photon polymerization. Microelectron. Eng. 2018, 194, $45-50$.

(44) Mann, S. A.; Sciacca, B.; Zhang, Y.; Wang, J.; Kontoleta, E.; Liu, H.; Garnett, E. C. Integrating Sphere Microscopy for Direct Absorption Measurements of Single Nanostructures. ACS Nano 2017, 11, 1412-1418. 